\title{
Primary malignant melanoma of the esophagus
}

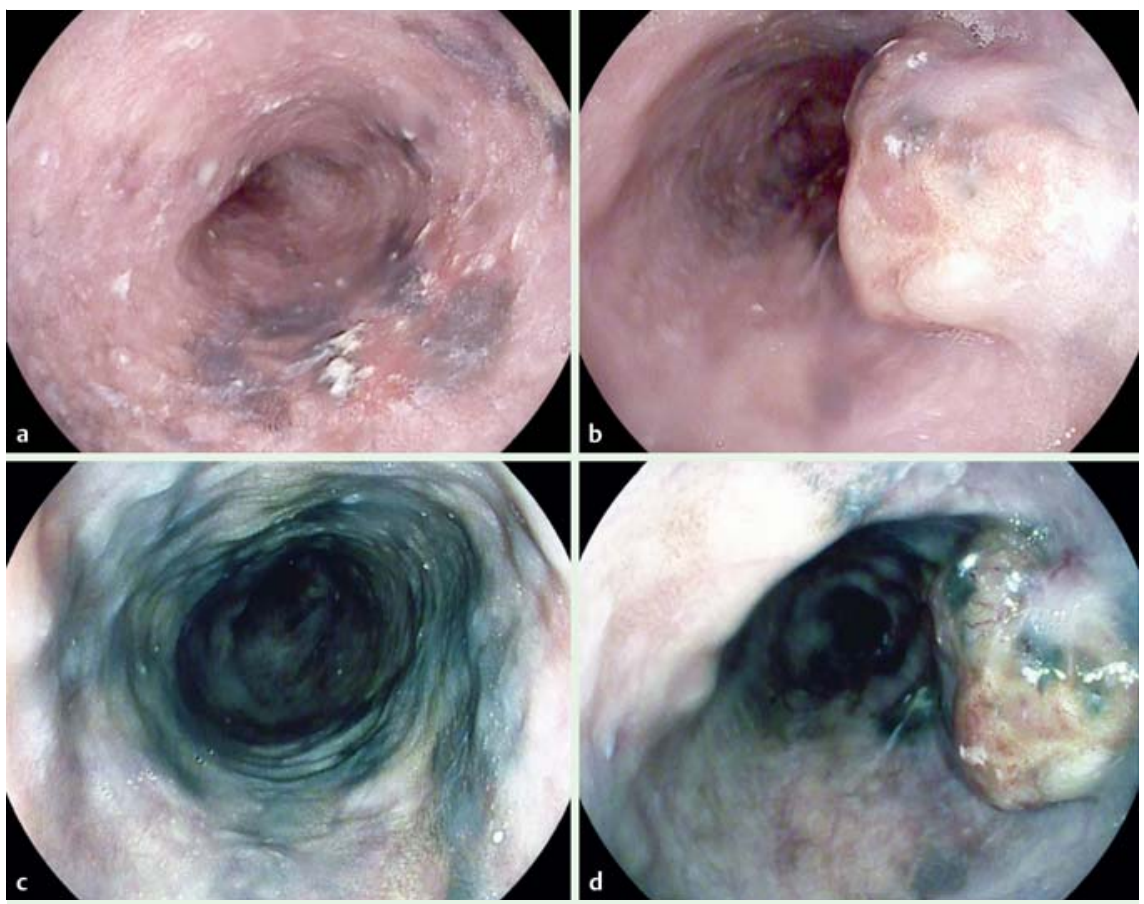

Fig. 1 Endoscopic appearance of mid to lower esophagus in a 75-year-old Caucasian man complaining of a 2-month history of dysphagia; a white-light endoscopy revealing background hyperpigmented mucosal plaques; b visible exophytic tumor; c, $\mathbf{d}$ background mucosal plaques and exophytic tumor using i-Scan technology (PENTAX HOYA, Japan).
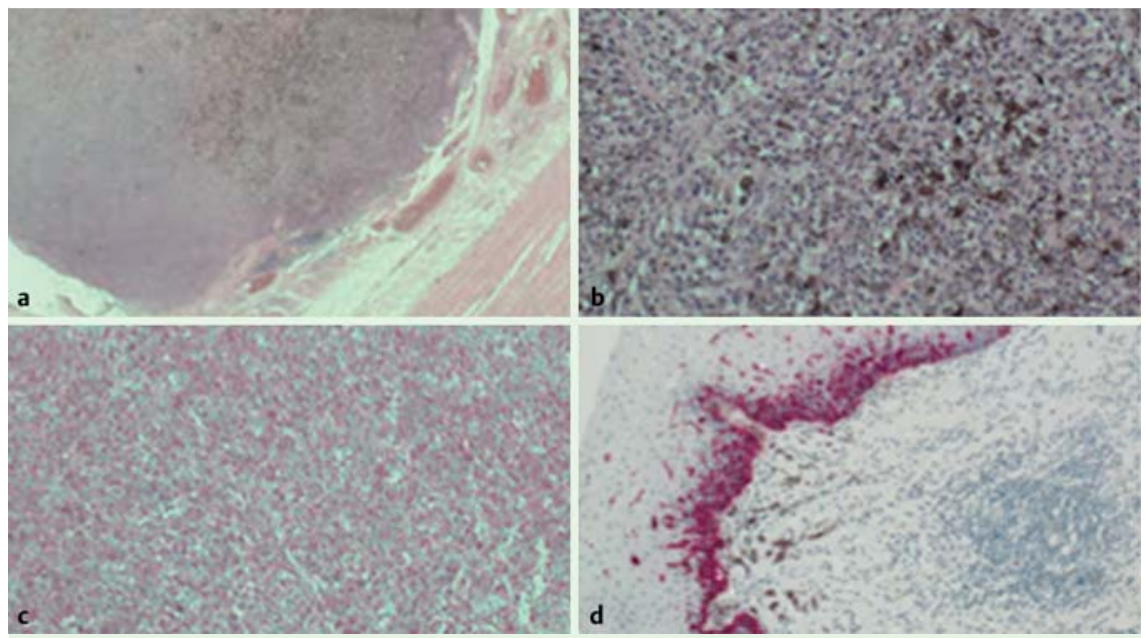

Fig. 2 a Histological section of the resected tumor specimen. b Histological section revealing abundant melanin. c Immunohistochemical staining demonstrating Melan A. d Human Melanoma Black-45 (HMB-45) highlighting the in situ component of the malignant melanoma.

A 75-year-old Caucasian man was referred to his local hospital complaining of a 2-month history of dysphagia. Esophagogastroduodenoscopy (EGD) demonstrated widespread mucosal hyperpigmented plaques throughout the esophagus. An exophytic esophageal lesion was also visualized in the mid to distal esophagus ( $\bullet$ Fig. 1). Biopsies of the exophytic lesion revealed findings consistent with primary malignant melanoma of the esophagus.
Positron emission tomography-computed tomography (PET-CT) demonstrated no evidence of metastases. A skin survey found no evidence of cutaneous melanoma. The patient underwent surgical resection with a three-stage esophagectomy. Histopathology of the esophagectomy specimen ( $\boldsymbol{O}$ Fig. $\mathbf{2}$ ) demonstrated a 70-mm tumor with abundant melanin and with a maximum depth of penetration of $9.5 \mathrm{~mm}$. Immunohistochemistry of tumor cells was positive for Human Melanoma Black-45 (HMB-45) ( $\bullet$ Fig. 2d), and Melan A ( $\bullet$ Fig. $\mathbf{2 c}$ ). Resection margins were free from malignant tissue as were 19 resected lymph nodes. TNM7 classification was T1bNOM0. Postoperative recovery was complicated by a superior mesenteric artery thrombus and ischemic bowel necessitating emergency smallbowel resection, right hemicolectomy, and defunctioning jejunostomy. Following 5 months of total parenteral nutrition, reversal of jejunostomy was done without complication. The patient is now living independently in the community, without parenteral nutrition.

Primary malignant melanoma of the esophagus accounts for less than $0.1 \%$ of all primary esophageal malignancies [1]. It presents similarly to other esophageal malignancies with most patients reporting dysphagia or weight loss [2].

Mean age at diagnosis is 60.5 years and it is twice as common in men as in women [3]. No other predisposing factors have been identified although benign esophageal melanosis is considered to be premalignant [3]. Prognosis is poor compared with other esophageal malignancies with 5 -year survival of just $2.2 \%$, one-sixth that of esophageal adenocarcinoma [4]. Surgical resection is the treatment of choice; however even with this intervention, mean survival is just 14.8 months with a 5 -year survival rate of only $4 \%$ [2]. Recent development of BRAF inhibitors has led to improvement in outcomes in the treatment of metastatic melanoma in patients with BRAF mutation; however its efficacy in primary malignant melanoma of the esophagus is unproven [5].

Adjuvant or neoadjuvant chemoradiotherapy has also been used but again is unproven [6]. In summary, we describe the case of a patient with primary malignant melanoma of the esophagus treated with a successful radical surgical resection. 
Endoscopy_UCTN_Code_CCL_1AB_2AC_3AB

Competing interests: None

Gabriel Wallis, Vinay Sehgal, Aiman Haider, John Bridgewater, Marco Novelli, Khaled Dawas, Rehan Haidry

University College London Hospitals NHS Foundation Trust, London, UK

\section{References}

1 Volpin E, Sauvanet A, Couvelard A et al. Primary malignant melanoma of the esophagus: a case report and review of the literature. Dis Esophagus 2002; 15: $244-249$

2 Morita FHA, Ribeiro U, Sallum RAA et al. Primary malignant melanoma of the esophagus: a rare and aggressive disease. World J Surg Oncol 2013; 11: 210

3 Iwanuma Y, Tomita N, Amano T et al. Status of primary malignant melanoma of the esophagus: clinical features, pathology, management and prognosis. J Gastroenterol 2012; 47: $21-28$

4 Leong QM, Kam JH. Primary malignant melanoma of the lower oesophagus presenting with dysphagia and upper gastrointestinal bleeding. Cases J 2008; 1: 28

5 Chapman PB, Hauschild A, Robert $C$ et al. Improved survival with vemurafenib in melanoma with BRAF V600E mutation. N Engl J Med 2011; 364: 2507-2516

6 Kelly J, Leader M, Broe P. Primary malignant melanoma of the oesophagus: a case report. J Med Case Rep 2007; 1: 50

\section{Bibliography}

Dol http://dx.doi.org/

10.1055/s-0034-1391126

Endoscopy 2015; 47: E81-E82

(c) Georg Thieme Verlag KC

Stuttgart · New York

ISSN 0013-726X

\section{Corresponding author}

\section{Gabriel Wallis, MBChB}

Department of Gastroenterology

University College London Hospitals NHS

Foundation Trust

235 Euston Road

London, NW1 2BU

UK

Fax: +44-20-34479217

gabrieljjwallis@gmail.com 